\title{
Critical appraisal of belinostat in the management of T-cell lymphoma - patient considerations
}

This article was published in the following Dove Press journal:

Blood and Lymphatic Cancer: Targets and Therapy

II September 2015

Number of times this article has been viewed

\author{
Andrew Bodiford' \\ Mahsa S Talbott' \\ Nishitha M Reddy² \\ 'Department of Pharmacy, ${ }^{2}$ Division \\ of Hematology and Oncology, \\ Department of Medicine, Vanderbilt \\ University Medical Center, Nashville, \\ TN, USA
}

\begin{abstract}
The histone deacetylase inhibitor (HDACi), belinostat, is an emerging and novel therapeutic option for patients diagnosed with relapsed/refractory peripheral T-cell lymphoma (PTCL). The PTCLs are comprised of multiple subtypes that occur in less than one per 100,000 cases in the USA. The incidence of these malignancies is rare, thus limited evidence is available. The most appropriate treatment modality has not been established. The most current recommended option is combination chemotherapy or enrollment in a clinical trial. T-cell lymphomas have emerged as a disease with marked epigenetic dysregulation. HDACi are an innovative and emerging medication class gaining increased attention in the treatment of T-cell lymphomas. There is a need to evaluate their potential place in the treatment of patients diagnosed with PTCL. Currently, the largest study evaluating belinostat use in this patient population is the BELIEF study. The BELIEF study is a single-arm, Phase II clinical trial, evaluating the use of belinostat in patients with refractory or relapsed PTCL. The primary outcome, objective response rate, was $26 \%$, with $11 \%$ achieving a complete response and $15 \%$ a partial response. This study presents a potential novel therapeutic option in the treatment of these patients. In this paper, we review therapeutic options for PTCL and present the recent data on the role of HDACi, specifically belinostat, in the treatment of patients with relapsed/refractory PTCL.
\end{abstract}

Keywords: peripheral T-cell lymphoma, novel agents, histone deacetylase inhibitor

\section{Peripheral T-cell lymphoma}

The peripheral T-cell lymphomas (PTCLs) are a rare, heterogeneous collection of disorders that occur in less than one per 100,000 cases in the USA, representing $10 \%-20 \%$ of all cases of non-Hodgkin's lymphoma. ${ }^{1,2}$ There are over 22 subtypes identified based upon the World Health Organization classification of lymphoid neoplasms. Regional differences exist, with the aggressive subtype, natural killer/T-cell lymphoma, being more common in Asian countries and rarely seen in European or North American countries. $^{3}$ The most common subtype worldwide is peripheral T-cell lymphoma, not otherwise specified (PTCL-NOS), which represents $29.5 \%$ of all PTCL diagnoses, along with angioimmunoblastic T-cell lymphoma (AITL) and anaplastic large cell lymphoma (ALCL) representing $18.5 \%$ and $12 \%$, respectively. The ALCL subtype is unique in that it is associated with anaplastic lymphoma kinase (ALK) amplification, which occurs in 50\% of all ALCL cases. PTCL-NOS and AITL typically affect older patients, while ALK+ ALCL affects younger patients and is associated with a much more favorable prognosis. ${ }^{4}$ Overall, PTCL has a very poor prognosis with a median overall survival (OS) of 9-42 months. To add to this dilemma, there are limited treatment options owning to the poor OS. ${ }^{5}$
Correspondence: Nishitha M Reddy Division of Hematology and Oncology, Department of Medicine, 3927 The Vanderbilt Clinic, Vanderbilt-Ingram Cancer Center, Nashville, TN 37232, USA $\mathrm{Tel}+\mathrm{I} 615936038 \mathrm{I}$

Fax +I 6159361812

Email nishitha.reddy@vanderbilt.edu 


\section{Treatment options}

The most appropriate and efficacious treatment option for PTCL has not been fully elucidated from clinical data due to the low incidence and poor outcomes observed with the available treatments for this aggressive lymphoma. The most promising results have been seen with combination chemotherapy, typically used in the treatment of B-cell lymphomas. In 2011, a meta-analysis evaluated the use of anthracyclinebased chemotherapy for the treatment of PTCLs. ${ }^{6} \mathrm{~A}$ total of 31 studies (13 prospective and 18 retrospective) involving 2,815 patients were included in this meta-analysis. The complete response (CR) rates ranged from $30 \%$ to $76 \%$ across the studies. The higher CR rates were observed in patients with ALCL. The 5 -year OS was $38.5 \%$ for all PTCL patients. The authors concluded that more robust treatment options are needed due to the poor 5-year OS observed.

More intensive combination chemotherapy regimens have been evaluated in an effort to improve upon the dismal OS rates associated with anthracycline-based regimens such as CHOP (cyclophosphamide, doxorubicin, vincristine, and prednisone). Other treatment options include ACVBP (doxorubicin, cyclophosphamide, vindesine, bleomycin, and prednisone), variations of $\mathrm{CHOP}$ such as $\mathrm{CHOP}-14$ and CHOP-21, and EPOCH (etoposide, vincristine, doxorubicin, cyclophosphamide, and prednisone). ${ }^{5}$ The median 5-year OS was still less than $40 \%$ after the inclusion of additional chemotherapy agents or adjustment of CHOP's administration schedule. Based on these results, there is a need to further elucidate more appropriate and efficacious options in the treatment of patients with PTCL.

\section{Autologous stem cell transplantation}

Autologous stem cell transplantation (ASCT) is a potential option in the treatment of patients with PTCL. The expected 3 - to 5 -year event-free survival ranges from $30 \%$ to $50 \%$, but there is controversy over the most appropriate time to initiate this treatment modality. ${ }^{7}$ Not all patients will respond to this therapy as evidenced by the event-free survival. Prior clinical trials determined that ASCT is only effective in chemosensitive disease and that this was one of the strongest predictors in terms of outcome. ${ }^{8}$ A recent Phase II clinical trial randomized patients to either combination chemotherapy followed by consolidation therapy with stem cell transplantation or six courses of CHOP-21 + alemtuzumab. ${ }^{9}$ Age had an impact on the treatment received; patients who were aged 60 years or younger were stratified to the transplantation arm, while patients aged $61-75$ years received combination chemotherapy. The 4-year OS was $49 \%$ for patients undergoing transplantation compared to $31 \%$ in patients receiving combination chemotherapy. There was also an improvement in 4-year progression-free survival (PFS) in favor of patients in the stem cell transplantation arm (44\% versus $26 \%)$. The authors determined that first-line allogeneic or autologous stem cell transplantation following induction chemotherapy improved OS rates in the younger patient population. In another study of 83 patients receiving ASCT in first remission, the estimated 3 -year PFS was $48 \% .{ }^{10}$ In the NORDIC lymphoma group trial, 166 untreated patients received ASCT in first remission, the 5-year PFS was $44 \%{ }^{11}$ Limitations of the nonrandomized trials and others evaluating the use of stem cell transplantation include small sample populations and the enrollment of patients with multiple subtypes of PTCL. It is difficult for the clinician to formulate definitive conclusions due to these limitations.

\section{Relapsed/refractory disease}

Chemotherapy, either single-agent or in combination with multiple agents, has not resulted in improved clinical outcomes for patients with PTCL. Resistance to chemotherapy treatment for PTCL can occur through the overexpression of P-glycoprotein, further decreasing the already dismal clinical outcomes observed in the treatment of PTCL. There is a need to evaluate novel treatment modalities to improve the OS and PFS in the treatment of PTCL. Agents previously investigated include gemcitabine, ${ }^{12}$ pralatrexate, ${ }^{13}$ allogeneic stem cell transplantation, ${ }^{14}$ monoclonal antibodies such as brentuximab, ${ }^{15}$ and the histone deacetylase inhibitors (HDACi). ${ }^{16}$

\section{Histone deacetylase inhibitors}

HDACi are an innovative and emerging medication class gaining increased attention in the treatment of T-cell lymphomas. Medications in this drug class include romidepsin, vorinostat, panobinostat, and belinostat. HDACi exert their activity through an alteration in the balance between acetylation and deacetylation, which plays a critical role in the proliferation of malignant cells in T-cell lymphomas. Levels of acetylation are balanced by histone acetyltransferase and HDAC. HDAC's major physiological activity is through the removal of acetyl groups from lysine located on histones causing a more compact chromatin. A compact chromatin results in transcriptional gene silencing and increased potential for unregulated cell proliferation. HDACi are pleiotropic drugs simultaneously targeting multiple signaling pathways essential for tumor cell 
survival. Belinostat and other HDACi allow hyperacetylation of these suppressed genes, resulting in cell growth arrest and/or apoptosis of malignant cells (Figure 1). A more detailed explanation of the molecular mechanism of action has been described previously. ${ }^{17}$

\section{Dosage and administration}

The maximum tolerated dose was investigated in a Phase I study in patients with various solid tumors. ${ }^{18}$ The dose range evaluated was $150-1,200 \mathrm{mg} / \mathrm{m}^{2} / \mathrm{d}$ intravenously (IV) over 30 minutes. Belinostat was administered on days $1-5$ of a 21-day cycle. The major dose-limiting toxicities were fatigue, nausea, vomiting, diarrhea, and atrial fibrillation, and the dose was established at $1,000 \mathrm{mg} / \mathrm{m}^{2} / \mathrm{d}$ based upon the toxicity profile observed in this Phase I trial. An oral formulation has been investigated in prior clinical investigations, and there is still a need to further evaluate the most appropriate place in therapy for oral belinostat. ${ }^{19}$

In 2014, the US Food and Drug Administration approved belinostat $1,000 \mathrm{mg} / \mathrm{m}^{2} / \mathrm{d}$ IV administered on days 1-5 every 21 days based upon clinical evidence, which will be discussed further in this review. ${ }^{20}$ The medication is administered over 30 minutes using a $0.22 \mu \mathrm{m}$ inline filter; if infusion site pain or other infusion-related adverse reactions occur, the infusion time can be increased to 45 minutes. Therapy with belinostat should be continued until the patient experiences disease progression or unacceptable toxicity. The dose adjustments are outlined in Table 1.

In patients with reduced UDP-glucuronyl transferase (UGT) 1A1 activity, the initial dose of belinostat should be reduced to $750 \mathrm{mg} / \mathrm{m}^{2} / \mathrm{d} .{ }^{21}$ Patients known to be homozygous for UGT1A1 *28 allele will have reduced UGT1A1 activity. The pharmacokinetic profile of belinostat was evaluated in patients with hepatocellular carcinoma to determine the impact of the UGT1A1 $* 28$ allele. The major route of metabolism did involve glucuronidation of belinostat through the UGT1A1 pathway. The presence of homozygous UGT1A1 *28 allele will result in lower glucuronidation activity for belinostat in comparison to patients with wild-type UGT1A1. Since belinostat is not metabolized as readily in patients known to be homozygous for UGT1A1 $* 28$ allele, it is recommended to provide a decreased initial belinostat dose. There are no dosage-adjustment recommendations available for patients with either hepatic or renal dysfunction. Recommendations for patients with moderate-to-severe hepatic impairment (total bilirubin $>1.5 \times$ upper limit of normal [ULN]) and/or creatinine clearance of $39 \mathrm{~mL} / \mathrm{min}$ or less are not available due to insufficient pharmacokinetic data available.

\section{Clinical trial experience}

The BELIEF trial evaluated the use of belinostat in patients with refractory or relapsed PTCL and remains the largest
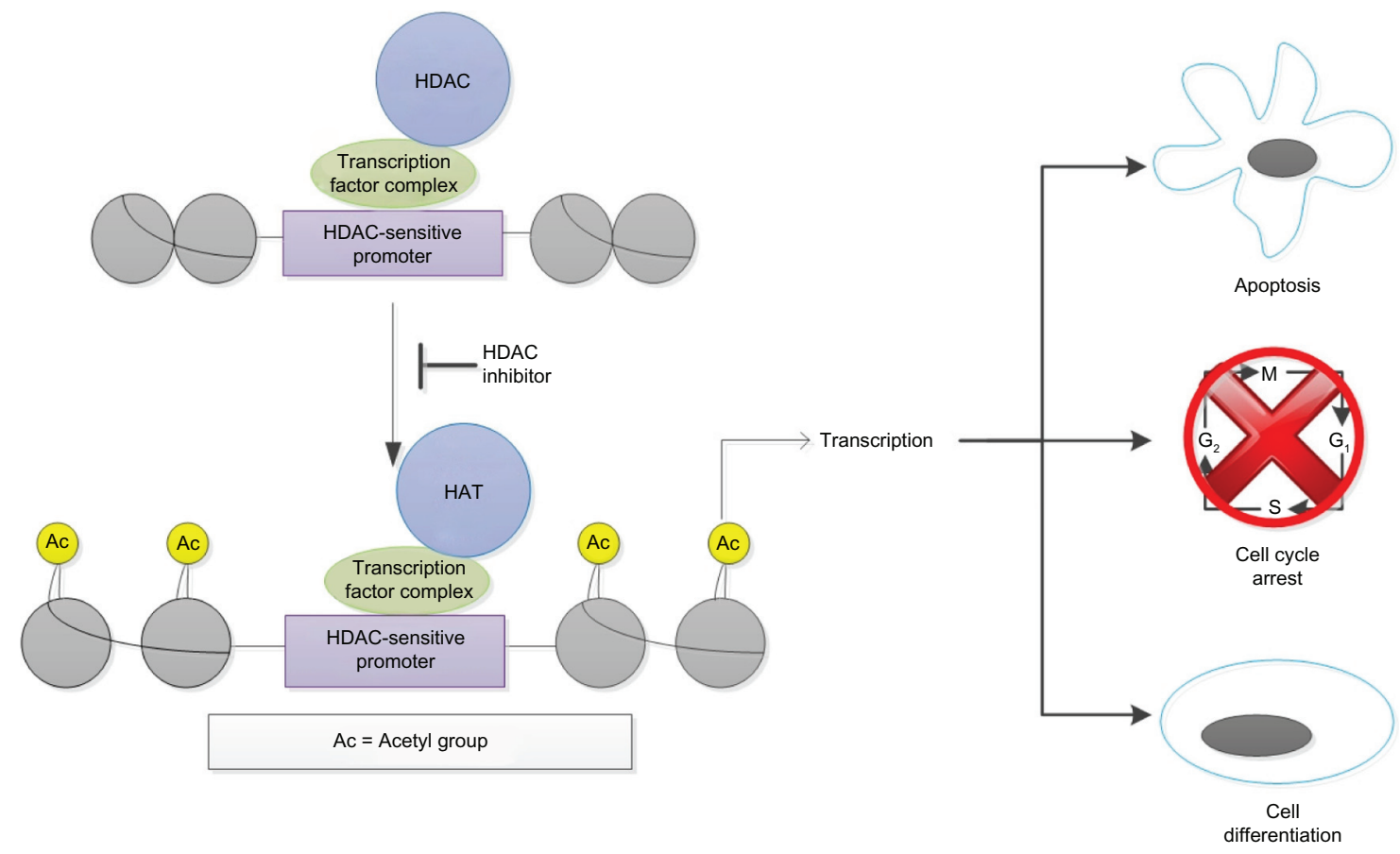

Figure I Pharmacology of histone deacetylase inhibitors.

Abbreviations: HDAC, histone deacetylase; HAT, histone acetyltransferase. 
Table I Dosing adjustments for toxicities

\begin{tabular}{ll}
\hline Hematologic toxicity & Recommendation \\
\hline Platelets $\geq 25,000 / \mathrm{mm}^{3}$ and nadir ANC $\geq 500 / \mathrm{mm}^{3}$ & No dosage adjustment necessary \\
Nadir ANC $<500 / \mathrm{mm}^{3}$ and any platelet count & Reduce dosage by $25 \%$ \\
Platelets $<25,000 / \mathrm{mm}^{3}$ and any nadir ANC & Reduce dosage by $25 \%$ \\
Recurrent ANC $<500 / \mathrm{mm}^{3}$ and/or recurrent nadir platelets & Discontinue belinostat treatment \\
$<25,000 / \mathrm{mm}^{3}$ after 2 subsequent dosage reductions & \\
Nonhematologic toxicity & Reduce dose by $25 \%$ \\
Any grade 3 or 4 toxicity & Provide supportive care and reduce the dose only if duration is \\
Grade 3 or 4 nausea, vomiting, or diarrhea & greater than 7 days, with appropriate supportive management \\
Recurrent grade 3 or 4 toxicity after 2 subsequent dosage reduction & Discontinue treatment \\
\hline
\end{tabular}

Note: ${ }^{a}$ All nonhematologic toxicities should be grade 2 or lower before reinitiating belinostat treatment at a lower dose.

Abbreviation: ANC, absolute neutrophil count.

clinical analysis of belinostat in this patient population. ${ }^{22}$ In this single-arm, Phase II clinical trial, patients were eligible for enrollment after failing at least one prior treatment. The primary study objective was to assess the safety and efficacy of single-agent belinostat in refractory or relapsed PTCL. Inclusion criteria included platelet counts of $50,000 / \mu \mathrm{L}$ or greater, no prior HDACi therapy, and relapse did not occur within 100 days following autologous or allogeneic hematopoietic stem cell transplantation. The primary endpoint was the objective response rate (ORR), and secondary endpoints were safety, time to response, PFS, duration of response, and OS. The response to treatment was also evaluated based upon the patient's PTCL subtype. Belinostat $1,000 \mathrm{mg} / \mathrm{m}^{2} \mathrm{IV}$ over 30 minutes was administered on days $1-5$ every 21 days, until the patient experienced disease progression or unacceptable toxicity. A total of 129 patients with relapsed or refractory PTCL received belinostat for a median of two cycles (range: 1-33). The majority of patients included were male (53\%), and the median age was 63 years. The median number of previous systemic therapies received was 2, and $93 \%$ of patients had received $\mathrm{CHOP} / \mathrm{CHOP}-$ like regimens. A smaller minority had previously undergone hematopoietic stem cell transplantation (23\%). There were 120 patients eligible for evaluation following central pathology review to confirm PTCL. Within this final patient population, seven patients died prior to first radiologic assessment, ten had clinical progression, five withdrew from the trial, and one was lost to follow-up. The ORR was $26 \%$ in the remaining patients, with $11 \%$ achieving a CR and $15 \%$ a partial response (PR). Stable disease was observed in $15 \%$, and $40 \%$ had progressive disease. The ORR in the three most common subtypes was $23 \%$ in patients with PTCL-NOS ( $\mathrm{n}=77$ ), $46 \%$ in AITL $(\mathrm{n}=22)$, and $15 \%$ in ALCL, ALK- tumors $(\mathrm{n}=13)$. Secondary outcomes are described in Table 2. The most common grade 3-4 treatment-related adverse reactions were thrombocytopenia (13\%), neutropenia (13\%), anemia $(10 \%)$, dyspnea $(6 \%)$, pneumonia $(6 \%)$, and fatigue $(5 \%)$. Based upon its adverse reaction profile, the authors concluded that belinostat is a potential therapeutic option for patients with low bone marrow reserve and/or thrombocytopenia. Belinostat also demonstrated a possible therapeutic advantage in the PTCL subtype, AITL, since patients diagnosed with this subtype experienced a higher ORR and a median PFS of 4.2 months compared to 1.6 months seen in all PTCL subtypes (Table 2).

A more recent Phase II clinical trial was conducted to evaluate the use of belinostat in patients with refractory/ relapsed PTCL or cutaneous T-cell lymphoma. ${ }^{23} \mathrm{~A}$ total of 53 patients diagnosed with PTCL or cutaneous T-cell lymphoma were included in this open-label, multicenter study. The median age was 65.9 years and the majority of the patients were Caucasian (68\%). All patients had failed at least one prior systemic therapy. Five patients $(20 \%)$ had undergone prior ASCT. The most common disease subtypes for the 24 patients with PTCL were PTCL-NOS (13 patients; 54.2\%), AITL (3 patients; $12.5 \%$ ), ALCL (3 patients; 12.5\%), and natural killer/T-cell lymphoma (3 patients; $12.5 \%$ ). A large proportion $(50 \%)$ had stage IV disease. Other pertinent inclusion criteria included adequate liver function defined as total bilirubin $\leq 1.5 \times \mathrm{ULN}$ or $\leq 3 \times \mathrm{ULN}$ if documented hepatic involvement with lymphoma, absolute neutrophil count $\geq 1.0 \times 10^{9} / \mathrm{L}$, platelet count $\geq 40 \times 10^{9} / \mathrm{L}$, and an estimated life expectancy $>3$ months. Patients who had received prior allogeneic stem cell

Table 2 Secondary outcomes

\begin{tabular}{ll}
\hline Outcomes & All patients $(\mathbf{N}=\mathbf{I} \mathbf{2 0})$ \\
\hline Median duration of response & 13.6 months \\
Median overall survival & 7.9 months \\
Median progression-free survival & 1.6 months \\
Median time to response & 5.6 weeks \\
\hline
\end{tabular}


transplantation were excluded. Belinostat $1,000 \mathrm{mg} / \mathrm{m}^{2}$ was IV administered on days 1-5 of a 21-day cycle. The primary outcome was ORR. Twenty-four patients with PTCL were included, and the median number of previous systemic therapies was 3 (range: 1-9). The median number of cycles received was 2 (range: $1-9)$, and the ORR was $25 \%$ (2 CR +4 PR) in patients with PTCL. The safety profile of belinostat was evaluated specifically in patients diagnosed with PTCL. There were five grade 3 adverse events and one grade 4 adverse event, including thrombocytopenia (grade 4), paralytic ileus, pneumonitis, maculopapular rash, macular rash, and cellulitis. There was one death due to ventricular fibrillation, possibly related to belinostat, that occurred 6 days following the last dose of the study medication in cycle 2 . An independent review determined that the arrhythmia was unlikely to be related to belinostat. The major treatment-related adverse reactions were nausea $(66.7 \% ; 16$ patients), constipation (37.5\%; 9 patients), and fatigue $(33.3 \% ; 8$ patients) within the PTCL population.

\section{Safety}

The safety profile of belinostat was previously evaluated in a Phase I dose-finding clinical trial. ${ }^{19}$ Cohorts of 3-6 patients with advanced solid tumors were enrolled sequentially into escalating dose steps. The dose was increased in 100\% increments until grade 2 toxicities were noted. After this, a sequential dose increase occurred such that the dose was increased by $50 \%$ until grade 3 toxicities were observed, and $33 \%$ thereafter. The most common adverse reactions, occurring in $\geq 15 \%$ of patients, were nausea, vomiting, fatigue, lethargy, constipation, flushing, and diarrhea. No hematological or grade 4 adverse reactions occurred at any dosing level. The maximum tolerated dose was determined to be $1,000 \mathrm{mg} / \mathrm{m}^{2}$.

The adverse reaction profile was similar in the BELIEF trial, which is one of the largest safety analyses of belinostat currently available. ${ }^{22}$ The most common grade $3 / 4$ adverse events observed in the 120 patients included were thrombocytopenia and neutropenia, occurring in $13 \%$ of the patients, respectively. Anemia (10\%), dyspnea (6\%), pneumonia ( $6 \%$ ), and fatigue $(5 \%)$ were the other common grade $3 / 4$ adverse reactions observed. Interestingly, belinostat was well tolerated in patients with platelet counts less than $100,000 / \mu \mathrm{L}$. This demonstrates a potential therapeutic benefit for belinostat in the treatment of patients with platelet counts less than $100,000 / \mu \mathrm{L}$. Belinostat was discontinued in $64 \%$ of patients due to progressive disease. The other major causes for medication discontinuation included death
$(11 \%)$, patient request $(8 \%)$, adverse events $(7 \%)$, and others $(4 \%)$.

Belinostat represents a potential therapeutic option for patients with thrombocytopenia, which is a class-wide adverse reaction, observed with HDACi (Table 3 ). The mechanism by which belinostat causes thrombocytopenia is not fully understood, but one theory has been explained previously. ${ }^{24}$ The HDACi decrease the transactivation function of GATA-1, an erythroid transcription factor, and this has the potential to affect posttranslational modification of GATA-1, leading to a delay in megakaryocyte maturation. Ultimately, thrombocytopenia can occur due to this interaction. Belinostat can be safely administered to patients with a platelet count $>25,000 / \mu \mathrm{L}$ without requiring any dosage adjustment, potentially demonstrating a novel therapeutic option in this patient population. Further studies are needed to determine this claim.

\section{Route of administration}

Currently, belinostat is only available as an IV infusion administered over 30 minutes. Patients receive belinostat on days 1-5 every 21 days, until disease progression or unacceptable toxicity occurs. Previous investigations have sought to determine the pharmacokinetic profile of an oral formulation, but there is no oral formulation of belinostat available at this time. The dosing and route of administration of other HDACi are outlined in Table 4.

\section{Future directions}

Belinostat as monotherapy has demonstrated promising results in patients with relapsed/refractory PTCL, which otherwise carries a very dismal prognosis. There are limited treatment options in this setting, and belinostat should be further evaluated to determine the most appropriate place for this novel treatment option. Future investigations should be directed at determining the viability of combination therapies due to belinostat's modest efficacy as a single agent. The

Table 3 Incidence of thrombocytopenia with HDACi

\begin{tabular}{ll}
\hline Medication & Incidence rate \\
\hline Romidepsin $^{16}$ & All grades: $17 \%-72 \%$ \\
& Grade 3/4: 36\% \\
Vorinostat $^{25,26}$ & All grades: $26 \%$ \\
& Grade 3/4: 6\% \\
Panobinostat $^{1}$ & All grades: $97 \%$ \\
Belinostat $^{22}$ & Grade 3/4: 67\% \\
& All grades: $16 \%$ \\
\hline
\end{tabular}

Abbreviation: $\mathrm{HDACi}$, histone deacetylase inhibitor. 
Table 4 Administration and available formulations

\begin{tabular}{|c|c|c|}
\hline Medication & $\begin{array}{l}\text { Available } \\
\text { routes }\end{array}$ & Administration \\
\hline Romidepsin ${ }^{16}$ & Intravenous & $\begin{array}{l}\text { Days I, } 8 \text {, and } 15 \text { on a } 28 \text {-day cycle } \\
\text { Administered over } 4 \text { hours }\end{array}$ \\
\hline Vorinostat ${ }^{25,26}$ & Oral & $\begin{array}{l}\text { Once daily until disease progression } \\
\text { or unacceptable toxicity }\end{array}$ \\
\hline Panobinostat $^{1}$ & Oral & $\begin{array}{l}\text { Every other day for three doses on } \\
\text { weeks I and } 2 \text { on a } 2 \text { I-day cycle }\end{array}$ \\
\hline Belinostat $^{22}$ & Intravenous & $\begin{array}{l}\text { Days } \mathrm{I}-5 \text { on a } 2 \mathrm{I} \text {-day cycle } \\
\text { Administered over } 30 \text { minutes }\end{array}$ \\
\hline
\end{tabular}

potential for an oral formulation of belinostat is quite promising given the potential for greater independence and ease of administration for the patient.

\section{Disclosure}

The authors report no conflicts of interest in this work.

\section{References}

1. Ellis L, Pan Y, Smyth GK, et al. Histone deacetylase inhibitor panobinostat induces clinical responses with associated alterations in gene expression profiles in cutaneous T-cell lymphoma. Clin Cancer Res. 2008;14(14):4500-4510.

2. Reimer P, Chawla S. Long-term complete remission with belinostat in a patient with chemotherapy refractory peripheral T-cell lymphoma. J Hematol Oncol. 2013;6:69.

3. Morton LM, Wang SS, Devesa SS, Hartge P, Weisenburger DD, Linet MS. Lymphoma incidence patterns by WHO subtype in the United States,1992-2001. Blood. 2006;107(1):265-276.

4. Hamadani M, Abu Kar SM, Usmani SZ, Savani BN, Ayala E, KharfanDabaja MA. Management of relapses after hematopoietic cell transplantation in T-cell non-Hodgkin lymphomas. Semin Hematol. 2014; 51(1):73-86.

5. Foss FM, Zinzani PL, Vose JM, Gascoyne RD, Rosen ST, Tobinai K. Peripheral T-cell lymphoma. Blood. 2011;117(25):6756-6767.

6. Abouyabis AN, Shenoy PJ, Sinha R, Flowers CR, Lechowicz MJ. A systematic review and meta-analysis of front-line anthracycline-based chemotherapy regimens for peripheral T-cell lymphoma. ISRN Hematol. 2011;2011:623924.

7. Reddy NM, Savani BN. Management of T-cell lymphomas: overcoming challenges and choosing the best treatment. Semin Hematol. 2014; 51(1): $1-4$.

8. Perrone G, Corradini P. Autologous stem cell transplantation for T-cell lymphomas. Semin Hematol. 2014;51(1):59-66.

9. Corradini P, Vitolo U, Rambaldi A, et al. Intensified chemo-immunotherapy with or without stem cell transplantation in newly diagnosed patients with peripheral T-cell lymphoma. Leukemia. 2014;28(9): 1885-1891.
10. Reimer P, Rudiger T, Geissinger E, et al. Autologous stem-cell transplantation as first-line therapy in peripheral T-cell lymphomas: results of a prospective multicenter study. J Clin Oncol. 2009;27(1):106-113.

11. d'Amore F, Relander T, Lauritzsen GF, et al. Up-front autologous stem-cell transplantation in peripheral T-cell lymphoma: NLG-T-01. J Clin Oncol. 2012;30(25):3093-3099.

12. Zinzani PL, Magagnoli M, Bendandi M, et al. Therapy with gemcitabine in pretreated peripheral T-cell lymphoma patients. Ann Oncol. 1998; 9(12):1351-1353.

13. O'Connor OA, Pro B, Pinter-Brown L, et al. Pralatrexate in patients with relapsed or refractory peripheral T-cell lymphoma: results from the pivotal PROPEL study. J Clin Oncol. 2011;29(9):1182-1189.

14. Schmitz N, Wu HS, Glass B. Allogeneic transplantation in T-cell lymphomas. Semin Hematol. 2014;51(1):67-72.

15. Horwitz SM, Advani RH, Bartlett NL, et al. Objective responses in relapsed T-cell lymphomas with single-agent brentuximab vedotin. Blood. 2014;123(20):3095-3100.

16. Piekarz RL, Frye R, Prince HM, et al. Phase 2 trial of romidepsin in patients with peripheral T-cell lymphoma. Blood. 2011;117(22): 5827-5834.

17. Xu WS, Parmigiani RB, Marks PA. Histone deacetylase inhibitors: molecular mechanisms of action. Oncogene. 2007;26(37):5541-5552.

18. Molife LR, de Bono JS. Belinostat: clinical applications in solid tumors and lymphoma. Expert Opin Investig Drugs. 2011;20(12):1723-1732.

19. Steele NL, Plumb JA, Vidal L, et al. Pharmacokinetic and pharmacodynamic properties of an oral formulation of the histone deacetylase inhibitor Belinostat (PXD101). Cancer Chemother Pharmacol. 2011;67(6): $1273-1279$.

20. Lee HZ, Kwitkowski VE, Del Valle PL, et al. FDA approval: belinostat for the treatment of patients with relapsed or refractory peripheral T-cell lymphoma. Clin Cancer Res. 2015;21(12):2666-2670.

21. Wang LZ, Ramirez J, Yeo W, et al. Glucuronidation by UGT1A1 is the dominant pathway of the metabolic disposition of Belinostat in liver cancer patients. PLoS One. 2013;8(1):e54522.

22. O'Connor OA, Horwitz S, Masszi T, et al. Belinostat in patients with relapsed or refractory peripheral T-cell lymphoma: results of the pivotal Phase II BELIEF (CLN-19) study. J Clin Oncol. 2015;33(23): 2492-2499.

23. Foss F, Advani R, Duvic M, et al. A Phase II trial of Belinostat (PXD101) in patients with relapsed or refractory peripheral or cutaneous T-cell lymphoma. Br J Haematol. 2015;168(6):811-819.

24. Matsuoka H, Unami A, Fujimura T, et al. Mechanisms of HDAC inhibitorinduced thrombocytopenia. Eur J Pharmacol. 2007;571(2-3):88-96.

25. Duvic M, Talpur R, Ni X, et al. Phase 2 trial of oral vorinostat (suberoylanilide hydroxamic acid, SAHA) for refractory cutaneous T-cell lymphoma (CTCL). Blood. 2007;109(1):31-39.

26. Olsen EA, Kim YH, Kuzel TM, et al. Phase IIb multicenter trial of vorinostat in patients with persistent, progressive, or treatment refractory cutaneous T-cell lymphoma. J Clin Oncol. 2007;25(21): 3109-3115.
Blood and Lymphatic Cancer: Targets and Therapy

\section{Publish your work in this journal}

Blood and Lymphatic Cancer: Targets and Therapy is an international, peer-reviewed, open access journal focusing on blood and lymphatic cancer research, identification of therapeutic targets and the optimal use of preventative and integrated treatment interventions to achieve improved outcomes, enhanced survival and quality of life for the

\section{Dovepress}

cancer patient. The manuscript management system is completely online and includes a very quick and fair peer-review system. Visit http://www.dovepress.com/testimonials.php to read real quotes from published authors. 